\title{
Exciton Percolation III. Stochastic and Coherent Migration in Binary and Ternary Random Lattices $\dagger$
}

\author{
P. ARgYrakis AND R. KOPELMAN \\ Department of Chemistry, The University of Michigan, Ann Arbor, \\ Michigan 48109, U.S.A.
}

(Received 22 February 1977, and in revised form 12 September 1977)

\begin{abstract}
We develop a method for calculating energy migration in random heterogeneous aggregates, with potential application to the primary process in photosynthetic units. A Monte Carlo technique is employed to study several types of random walk motion in a random binary lattice. Our computations include 2 and 3 dimensional lattices of different topology and employ correlated steps with a Gaussian distribution of directional memory. The effects of the characteristics of the motion and its parameters are displayed and discussed. The lower threshold for efficient visitation by the walker is given by the critical percolation concentration. However, a higher threshold is found in the case of coherent motion. This new "turning point" appears to play an important role in the process of exciton transport. The exciton percolation formalism is utilized, giving results for ternary random lattices where the third component is very dilute and acts as a sensor. The results are applied to a system representing the ${ }^{1} B_{2 u}$ naphthalene exciton dynamics in an isotopic and chemically mixed crystal, which by itself is supposed to mimic the exciton transport in the photosynthetic units of green plants. Physically reasonable parameters, trends and limits are discussed. Also, an analytical solution is derived and tested for a physically reasonable limit of semicoherent motion in a perfect lattice. The ramification of this work on bioexciton transfer is discussed, especially concerning the light harvesting units in green plants. It leads to a simple minded model that rationalized the ratio of antenna to active-center molecules. Our most important result is that incoherent exciton transfer, i.e. simple random walk, is the most efficient process for significantly heterogeneous aggregates.
\end{abstract}

\section{Introduction}

Molecular energy transfer in biological systems is widespread. Excitonic type energy transfer, i.e. excitation transfer similar to that in molecular crystals (and possibly liquids), has been claimed for many and various

†Supported by NSF Grant DMR75-07832 A01 and NIH Grant NS08115-08. 
biological systems, including the "integrated activity involved in mental proccsscs" and mitochondrial respiration (Ressler, 1969), cardiac and other muscle action, i.e. Davydov (1973), Davydov \& Kislukha (1976). The most studied systems are the photosynthetic units of green plants, i.e. Robinson (1967), Pearlstein, (1967), Sauer (1975), Knox (1975), Katz, (1976). While the investigations dealing with the excitation dynamics usually assume an ordered lattice of chromophores, i.e. of chlorophyll-a (Robinson, 1967; Pearlstein, 1967; Montroll, 1969), the actual light harvesting aggregate is usually believed to be disordered in various ways, due to different possible environments (proteins, water molecules, other chromophores etc.). Proposed models have been discussed recently (Sauer, 1975; Katz, 1976; Caple et al., 1976; Tien, 1976). Borisov, i.e. Borisov \& Godik (1973), has discussed transfer in heterogeneous units which, however, are neatly arranged energy "funnels". The importance of exciton transfer in heterogeneous, substantially disordered, biological aggregates has just been discussed for the photosystem I and II quantum yield, fluorescence lifetime and their temperature dependence by Swenberg, Dominijani \& Geacintov (1976), following our preliminary suggestions (Kopelman et al., 1975b).

The random walk computer calculations used by Swenberg et al. were of limited nature, utilizing only very small lattices $(5 \times 5$ sites $)$, and they did not actually utilize percolation theory (Broadbent \& Hammersley, 1957) or the exciton percolation formalism (Hoshen \& Kopelman, 1976). We solve here a more general problem with a wider range of solutions and a much higher precision, using computer simulation on statistically large enough lattices, with various topologies, using analytical formulae to get the ternary lattice results from binary lattice computations. A preliminary report has been given earlier (Kopelman, 1976c). There has also been wide discussion on the nature of the exciton energy transfer, whether it is coherent or incoherent (Knox, 1975). If it is completely incoherent, one is lead to a simple random walk model (Montroll, 1969). If it is partially coherent, Montroll's model must be modified in some way (Pearlstein, 1972; also see later). Much of this effort was aimed at elucidating the possible effects of such coherent transfer on the role of energy transfer in a photosynthetic system. Obviously, of major significance is the fact that the excitation transfer in a photosynthetic unit seems to be mostly over in picoseconds, a time short enough to prevent major losses via fluorescence, the radiative emission time scale being of the order of nanoseconds (Sauer, 1975; Katz, 1976). It is usually believed that "coherent" transfer is faster, and thus more efficient, than incoherent, i.e. simple random walk type transfer. We show here that, while this is true for ordered and nearly ordered lattices, quite the opposite is expected for disordered lattices (and 
aggregates). We thus believe that our calculations given here are not only of importance to excitonic transfer, but emphasize the advantages of simple stochastic motion over that of "coherent" motion whenever such a process takes place in a non-isotropic, non-homogeneous medium. Possible applications include the motions of ions through membranes (Tasaki, 1976; Abood, 1976). We notice here that while we assume in our work random substitutional disorder, for mathematical convenience, the qualitative aspects of our results should hold as well for "correlated" disorder, as has been shown for binary lattices (Muller-Krumbhaar, 1974; Drucker \& Ross, 1974).

There has been much interest in random walk problems on lattices (Montroll, 1964; Montroll \& Weiss, 1965; Montroll, 1969; LakatosLindenberg \& Shuler, 1971; Lindenberg, 1974) and their relation to the transport of excitation in photosynthetic units (Montroll, 1969; Knox, 1975; Sauer, 1975; Olson, Ke \& Thompson, 1976). Much recent work has been concerned with the effects of exciton coherence (Kenkre \& Knox, 1976; Silbey, 1976; Pearlstein, 1972; Hemenger, Pearlstein \& Lakatos-Lindenberg, 1972; Lakatos-Lindenberg, Hemenger \& Pearlstein, 1972; Hemenger, Lakatos-Lindenberg \& Pearlstein, 1974) including partial coherence combined with random walk (Pearlstein, 1972; Hemenger et al., 1972; LakatosLindenberg et al., 1972; Hemenger et al., 1974). Also recently (Hoshen \& Kopelman, 1976a; Argyrakis \& Kopelman, 1977; Swenberg, Dominijanni \& Geacintov, 1976; Kopelman, 1976a, b, c), emphasis has been given to models based on random heterogeneous lattices, containing two or more major components, i.e. chl. $a$ and chl. $b$, in addition to the minor component playing the role of both ultimate trap and active center, i.e. $P$ 700. Essential for the energy transport in the random heterogeneous aggregate is the presence of a microscopically connected quasilattice consisting only of sites that contain the major component with the lowest excitation energy. Only the ultimate trap ("supertrap") is allowed to have an even lower excitation energy, in this model of exciton percolation (Hoshen \& Kopelman, 1976a; Kopelman, Monberg and Ochs, 1977a, $b$, Hoshen, Kopelman \& Monberg, 1978; Kopelman et al., 1975a, b; Kopelman, 1976a, b, c). It is usually assumed that an ensemble of small random lattices, i.e. photosynthetic units, can be simulated best by a large random lattice with cyclic boundary conditions (Kopelman, 1976b). Such a simulation is also suitable for the exciton percolation exhibited in mixed molecular crystals, including those alloyed in proportions required to mimic the energy transport in the primary process of photosynthesis (Kopelman, et al., 1975a; b; Kopelman, 1976a, b, c). We note here that the exciton percolation probability ("Registration Probability") $P$ which we calculate in this work is the same as the quantum 
yield of excitation trapping, which for photosynthesis is (Borisov \& Godik, 1973):

$$
\Phi_{p h}=K_{p h} /\left(K_{\Sigma}+K_{p h}\right),
$$

where $K_{p h}$ is the rate constant of excitation deactivation by the photosynthetic reaction centers, and $K_{\Sigma}$ is the rate constant of the "overall trivial deactivation processes" (Borisov \& Godik, 1973).

The random walk theories that have been developed during the last fifteen years (Montroll, 1964; Montroll \& Weiss, 1965; Montroll, 1969; Lakatos-Lindenberg \& Shuler, 1971; Lindenberg, 1974) involve lattices with only one major component. They have been successful in deriving exact analytical expressions for several lattice characteristics. However, there is no such theory, to date, to treat in a similar fashion the full range of binary or more complex lattices. On the other hand, percolation theory has been suggested as an aid to the investigation of random walk type motion in such systems (Hoshen \& Kopelman, 1976; Argyrakis \& Kopelman, 1977; Kopelman, 1976c).

In the present study a general Monte Carlo technique is employed on a finite lattice made of two types of sites, $A$ and $B$ sites. The only other similar study on binary systems has been recently reported by W. W. Brandt (Brandt, 1975). His technique is similar to ours and uses open and closed sites (like our $A$ and $B$ ) for estimating effective diffusion coefficients of a migrating fluid. The size of his lattice is considerably smaller than ours and his application is limited to simple random walk. We examine twodimensional topologies, such as square and triangular, and a threedimensional one (simple cubic). The motion is treated as simply random, i.e. steps of length one, or partially coherent (steps of varying length). We propose that our derived results hold for an infinite lattice as well, as the lattice employed is fairly large (of the order of $10^{5}$ to $10^{6}$ sites) and the total activity on the lattice, such as the number of steps of the random walker, is of the same or lower magnitude (Pearlstein, 1972; Hemenger et al., 1972; Lakatos-Lindenberg et al., 1972; Hemenger et al., 1974).

Our exciton percolation studies (paper I [Hoshen \& Kopelman, 1976a)]: general theory, paper II [Kopelman et al., 1977b]: supertransfer experiments) continue here, emphasizing time limited coherent and incoherent motion. In section 2 the details of the computational method are explained. The results are expressed as the number of sites visited by the "random" walker, for a specific number of steps, under different sets of conditions. These calculations are independent of percolation theory but derive their impetus from it (Hoshen \& Kopelman, 1976a; Argyrakis \& Kopelman, 1977). In section 3 we use the recently developed formalism of exciton percolation 
and apply it to the ${ }^{1} B_{2 u}$ naphthalene system, which is supposed to mimic exciton transfer in natural systems (Kopelman, 1976a, b,c). For this system we show the importance of the size, topology and relative magnitudes of the molecular interactions and the effect of varying the correlation (memory) between steps. We believe this to be the key to the investigation of coherence in molecular systems (Argyrakis \& Kopelman, 1977). The effects of the various parameters are discussed in section 4 . The most significant result is that, in addition to the critical percolation concentration of exciton carriers below which no direct energy migration takes place, there is also a "turning point", at a higher concentration, below which partial correlation (coherence) of the motion has a negative effect on the energy transport. This result for heterogeneous systems is directly the opposite from the wellknown result for homogeneous systems (with no or little concentration of scatterers), for which the transport becomes more efficient with added correlation (coherence). We also give an analytical result for a physically reasonable limit of semicoherent motion in a perfect lattice (Appendix B).

\section{Random Walk Computations}

A binary square lattice made of $A$ and $B$ sites is simulated and kept in the computer memory for the 2-dim. topologies. Several such layers are used for the 3-dim. ones. The standard IBM subroutine RANDU is used (in its more efficient variant, URAND, of the Michigan Terminal System) to decide whether the site in question is $A$ or $B$ according to the given concentration of $A, C_{A}$ where $C_{\mathrm{A}}$ goes from 0.0 to 1.0. Therefore, the lattice is built in a completely random fashion. The dimension of the lattice $N$ is an adjustable parameter, typically being a quarter or one half million sites. The number of $A$ sites is therefore $N_{A}=C_{A} N$, where $N$ is the total number of sites. The origin of motion is randomly chosen and located on a specific lattice site. Then the random walker starts moving to adjacent sites. The direction is again chosen at random, say from the four available choices of the nearest neighbors (when a square lattice symmetry with nearest neighbor jumps is considered). After each step all memory is lost and the process repeats itself anew for a total number of steps, $t$. The programming allows motion only on the terrain of $A$ sites but no access is allowed onto $B$ sites. Thus, $N_{A}$ is the upper limit for the number of sites that can be visited. This is possible only for high $C_{A}$, i.e. when $C_{A} \rightarrow 1.0$, where all $A$ sites in the lattice are connected. However, as $C_{A}$ decreases the number of available $A$ sites decreases even faster because now clusters of $A$ start to appear. A cluster of $A$ sites is a set of connected $A$ sites completely surrounded by $B$ sites. Since all $B$ sites are non-accessible, all but one $A$ 
clusters are also non-accessible to a given walker. Again, the smaller $C_{A}$, the smaller is the available number of $A$ sites. We focus on the largest cluster available in the crystal, the so called maxicluster. Its size is strongly concentration dependent (Hoshen \& Kopelman, 1976b).

What we compute is the number $n_{m}$ of distinct sites visited at least once during a random walk on the maxicluster, where the maxicluster consists of $m$ sites of type $A$. From percolation theory the general cluster distribution is now known (Hoshen \& Kopelman, 1976b) and it can be shown (Hoshen \& Kopelman, 1976b; Kopelman et al., 1977a) that the size of the maxicluster increases dramatically at the critical site percolation concentration $C_{C}^{s}$, while it drops to small values below it. It is obvious that well below $C_{C}^{s}, n_{m}$ will usually be about equal to $m$, a number we can predict from percolation theory. Therefore, we focus on the region $C_{A} \geqslant C_{C}^{s}$.

In order to avoid interruptions in the process at the finite ends of the lattice, boundary conditions are employed (i.e. cyclic) so that the lattice repeats itself indefinitely. However, the walker rarely "hits" the boundary, for most computation (see Discussion). We can now extend the random walk process to include direct hops to next-nearest-neighbors, next-nextnearest-neighbors, etc. The program gives the option of assigning different hopping probabilities to different kinds of jumps. This probability will depend on the specific application at hand. As the number of the available neighboring sites varies, the cluster distribution also varies and this directly affects $n_{m}$. In Fig. 1 we show the case for lattices with the following topology: square (four neighbors), triangular (six neighbors), and square $(1,2)$ (eight neighbors). While the exact values of $n_{m}$, at high and intermediate $C_{A}$, are model dependent (section 3), it is still true in general that the larger the co-ordination number, the larger is $n_{m}$, and for each case the fall-off point is where $C_{c}^{s}$ has been previously established (Hoshen \& Kopelman, 1976a, b, $c$ Argyrakis \& Kopelman, 1977; Hoshen et al., 1977), i.e. 0.593 for the square lattice, 0.500 for the triangular one and 0.41 for the square $(1,2)$ lattice.

We have assumed up to now that all memory is lost after $l=1$ jumps. We call $l$ the coherency parameter or just coherency. We now allow the random walker to make $l$ jumps in a row in the same direction, which is similar but not equal to making a jump of length $l$. Even of greater interest is the case where $l$ is the average or most probable value of a distribution, rather than a constant. This idea has been utilized recently (Lakatos-Lindenberg \& Shuler, 1971) and solved analytically, but only for a one-dimensional, pure (one-component) lattice, using exponentially distributed jumps. In our case the coherency of the given jump is derived from a Gaussian distribution where we define the mean and the standard 


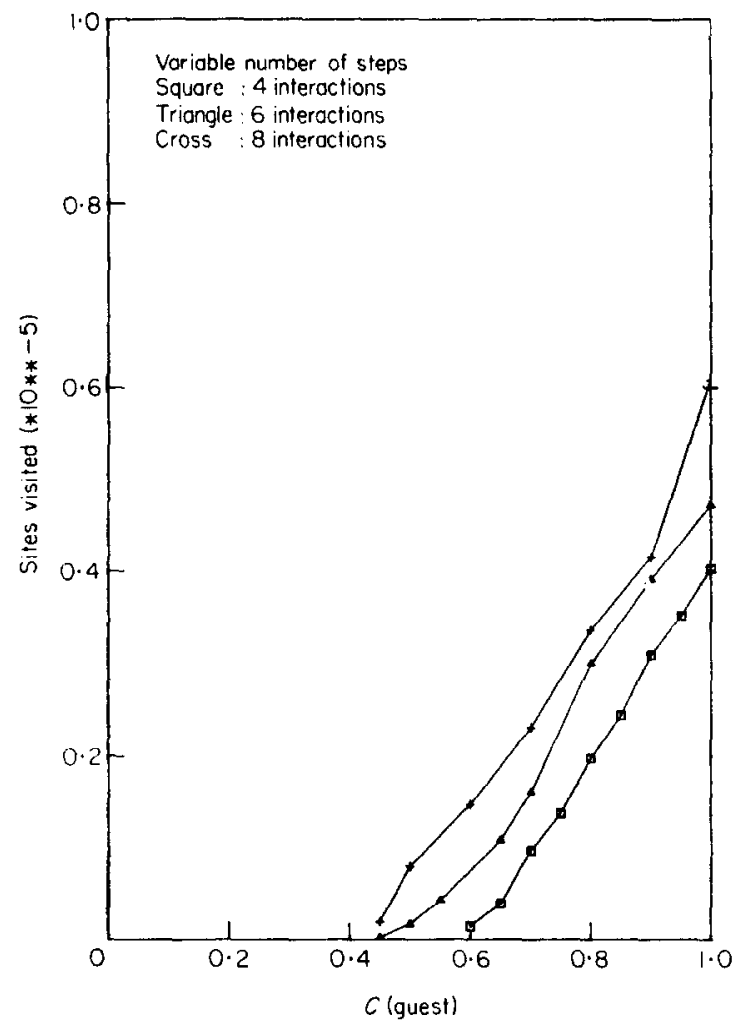

FIG. 1. The number of distinct sites visited $\left(n_{m}\right)$ vs. guest concentration $\left(C_{G}\right)$, for square, triangular and square $(1,2)$ topologies. The number of steps $(t)$ is $172000 \times C_{G}$. The lattice size is $250000(=500 \times 500)$, with cyclic boundary conditions. Note that unequal jump probabilities were used, i.e. $p: p^{\prime}: p^{n}=10: 5: 2$, where $p$ represents the four nearest neighbor jumps, $p^{\prime}$ the next two and $p^{\prime \prime}$ the last two.

deviation of the lineshape. As in the case of $l=1$, at the end of these $l$ hops all memory is lost, while within them it is perfectly retained, i.e. all successive $l$ jumps are completely correlated, directionally. We note, however, that in our model this correlation is completely broken as soon as the hopping path is blocked by a $B$ site.

\section{Applications}

The quantities $n_{m}$ of the previous section can now be applied to particular molecular aggregates. One can compare the types of random motion described earlier and examine their characteristics, such as visitation 
efficiency, concentration dependence, etc. We have chosen as a guide the isotopic mixed naphthalene system (Kopelman et al., 1977b), where the $A$ sites are occupied by $\mathrm{C}_{10} \mathrm{H}_{8}$ and the $B$ sites by $\mathrm{C}_{10} \mathrm{D}_{8}$. This is an excellent model system (Kopelman, 1976b, c). The random motion could describe an exciton migration process in this system. We initially use the total number of steps, $t$, derived as follows. The lifetime of the first excited singlet state is (El-Kareh \& Wolf, 1968; Uchida \& Tomura, 1974; Kohler, Schmid \& Wolf, 1976) about $100 \mathrm{~ns}$. The jump time is calculated (Hanson, 1975) from $t_{j}=(4 M)^{-1}$, where $M$ is the absolute pairwise interaction (Hanson, 1970; Hong \& Kopelman, 1971; Ochs \& Kopelman, 1977; Kopelman, 1975) in hertz. For $M=17 \mathrm{~cm}^{-1}$ we get $t_{j}=0.5 \mathrm{ps}$. The total number of steps equals that of jumps: $t \cong 100 \mathrm{~ns} / 0.5 \mathrm{ps}=2 \times 10^{5}$ steps.

At this point, we notice that the parameter $n_{m}$, the number of distinct sites visited, monitors the excitonic migration rather indirectly. A more direct way is to introduce a set of registers $S$ in the lattice, which "sense" the random walker every time it arrives at such an $S$ site and register all such visits accordingly. We are actually just saying that it is difficult to experimentally measure the parameter $n_{m}$, while doping the crystal with an impurity is an easy way to introduce the set $S$ of sensors. The $A$ and $S$ sites together now make up the "guest" quasilattice (i.e. its various clusters). The $B$ sites make up the "host" quasilattice.

Let the concentration of sensors be $C_{S}$. Then the total number of sensors in the lattice is $Z=C_{S} N$, where $N$ is the total number of lattice sites. It has been shown (Hoshen $\&$ Kopelman, 1976a, $c$ ) that the probability $F_{n}$ of having at least one sensor included in a set of sites is:

$$
F_{n}=1-\left(1-\frac{n_{m}}{G}\right) z \quad \text { iff } Z \ll G,
$$

where $G$ is the total number of "guest" ( $A$ and $S$ ) sites. Furthermore, if the largest cluster is of size $m$, as assumed earlier, then $\bar{P}_{\infty} \equiv m / G$ is the probability that the random walker starts its motion inside this cluster. Once a walker is on the maxicluster (Hoshen \& Kopelman, 1976a, c), its probability $\bar{F}_{m}$ of registering on a sensor is that of stepping on any such sensor. This is ture for our case, where $Z \ll G$, if sensor saturation can be ignored. Furthermore, we assume that multiple registrations can be neglected. This is a good assumption for $Z \ll G$ and it is certainly true for a sensor registration efficiency of unity $(\gamma=1)$, which we imply here. Hence the registration probability $\bar{F}_{m}$ is

$$
\bar{F}_{m}=F_{n} \quad \text { iff } Z \ll G \text { and } \gamma=1,
$$

and thus the total probability of a walker being registered by a sensor 
inside the maxicluster is:

$$
P=\bar{P}_{\infty} F_{n} \quad \text { iff } \gamma=1, C_{G}>C_{C}^{s} \text { and } Z \ll G .
$$

Notice that, for $C_{G} \gtrsim C_{C}^{s}$, the cluster size $m$ is of the order of $G$. Thus $t$ should be smaller than $G$, to avoid saturation in time, i.e. avoiding "guaranteed" registration for $Z \geqslant 1$. Such saturation is a trivial case of "supertransfer" (Hoshen \& Kopelman, 1976a; Kopelman et al., 1977b), leading to $n_{m} \cong m$ and thus to $F_{n} \cong 1$ and $P \cong \bar{P}_{\infty}$. We have shown before (Hoshen \& Kopelman, 1976a; Kopelman et al., 1977b) that for $C_{G}>C_{C}^{s}$ the contributions of the miniclusters to exciton migration are negligible. Thus exciton migration is given by equation (3), with the assumption of $\gamma=1$. For $\gamma<1$ we have suggested simple correction factors (Hoshen \& Kopelman, 1976a; Kopelman, 1976c). As $Z$ increases, $F_{n}$ approaches $F_{m}$ (where $F_{m}$ is defined by equation 1 with $n_{m}=m$ ), i.e. becomes a constant for a particular $C_{G}$ concentration (the limit of "supertransfer") (Kopelman, 1976c). The supertransfer limit for naphthalene has been experimentally established (Kopelman et al., 1977b; Kopelman et al., 1975a) at $C_{S} \sim 1 \times 10^{-3}$ mole fraction, as is easily predicted in this work. We therefore investigate the region where $C_{S}<1 \times 10^{-3}$, i.e. where $Z \ll G$. Also notice that we have (Hoshen \& Kopelman, 1976a) a formulation for $\bar{F}_{m}$ which depends on $n_{m}, G$ and $Z$ only, i.e. we do not need to perform computer simulations on lattices that actually include sensors. Thus, just from binary system simulations we can estimate the ternary system probabilities. Note that $n_{m}$ is defined for $Z=0$.

Some results are demonstrated by the sample calculations given in Fig. 2-8. In Fig. 2 we have plotted $P$ versus $C_{G}$. The topology is that of nearest neighbors in a square lattice (co-ordination number 4 ) with lattice size $N=500 \times 500=250000$. The total number of steps is $t=200000$ and equals the total number of jumps, as the number of correlated jumps is $l=1$. In paper I (Hoshen \& Kopelman, 1976a) we gave curves describing the behavior for $C_{S}$ up to $1 \times 10^{-2}$ (but $C_{S} \leqslant C_{G}$ ), the latter giving the same curve as found from percolation theory (Hoshen et al., 1977; Kopelman, 1976c). Notice that it provides a $C_{C}^{s}$ value of about 0.59 . As we lower $C_{S}$ we get the family of curves in Fig. 2 . At any $C_{G}$ concentration the total probability $P$ decreases as $C_{S}$ decreases (see appendix A). Also, $P$ practically vanishes below $C_{C}^{s}$, as discussed in section 2 . This limiting behavior is achieved by ignoring the contributions of the smaller clusters (Kopelman, 1976c), which become important for $C_{G} \leqslant C_{C}^{s}$. In Fig. 3 a plot is shown of $P$ vs. $C_{G}$ for a constant sensor concentration $C_{S}=2.5 \times 10^{-5}$, but for several different $t$ values. Again, as $t$ increases, $P$ also increases. In Fig. 4 the data is the same as in Fig. 2, with the addition 


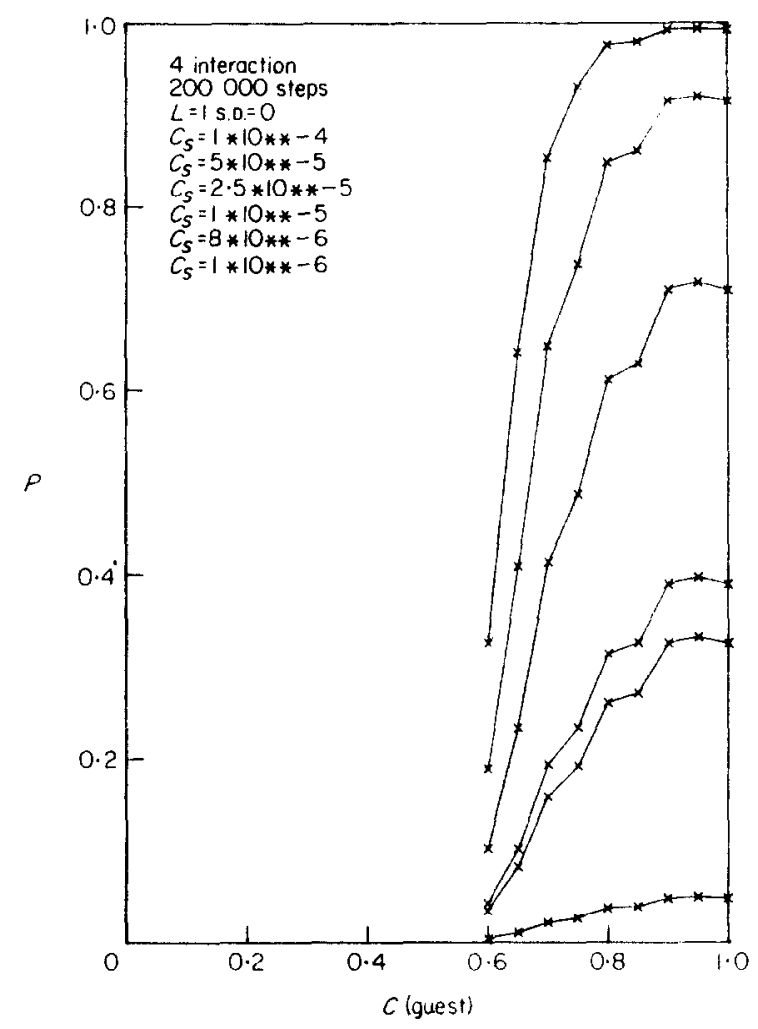

FIG. 2. The total probability $P$ [equation (3)] of a random walker being registered by a sensor site vs. the guest concentration $C_{G}$ (mole fraction) for six different sensor concentrations. The topology is that of the square lattice (four nearest neighbor interactions). The step length $(L)$ here is $l=1$, the lattice size $(N)$ is 250,000 and the number of steps $(t)$ is 200,000 . The sensor concentration $C_{S}$ (mole fraction) is the variable parameter $\left(10^{-6}\right.$ to $10^{-4}$, bottom to top curve, respectively). Note that while the roughness of each curve is due to computational fluctuations, the values for any given $C_{G}$ are not so afflicted. For higher $C_{S}$ values, see Fig. 3 of Hoshen \& Kopelman (1976a) and Kopelman (1976c).

of next-nearest neighbor jumps. These jumps were given one half the probability of the nearest neighbor jumps (and each jump also consumes two "steps"--see below). We were guided in this choice by the ratio of interactions in the ${ }^{1} B_{2 u}$ naphthalene system, where the added interaction may correspond to the $b$-axis "translational" interaction, besides the $\pm \frac{1}{2}(a \pm b)$ interaction (Hanson, 1970; Hong \& Kopelman, 1971; Ochs \& Kopelman, 1977; Kopelman, 1975). In Fig. 5 we add the next-next nearest neighbor jumps where the probability is one-fifth compared to the nearest neighbor jumps. This may correspond to the $a$-axis "translational" interaction 


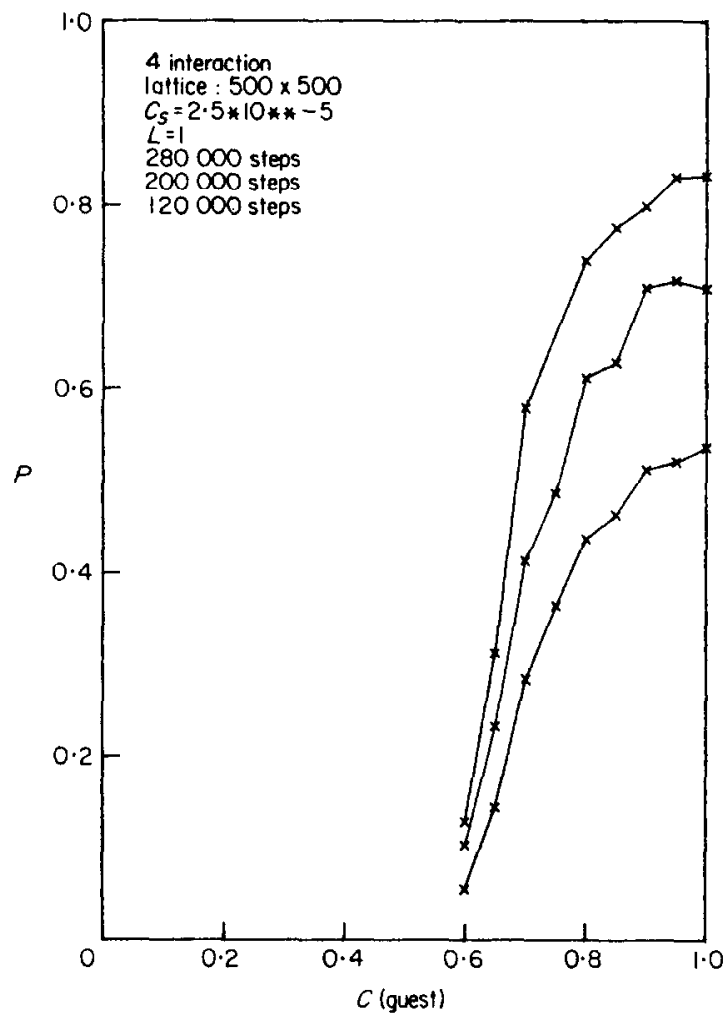

FIG. 3. Registration probability $P$ vs. guest concentration $C_{0}$. This is the same as Fig. 2 , except that here the parameter is the number of random walk steps $t(120000-280000$, bottom to top curve, respectively), and the sensor concentration $C_{S}$ is fixed at $2.5 \times 10^{-5}$.

(Hanson, 1970; Hong \& Kopelman, 1971; Ochs \& Kopelman, 1977; Kopelman, 1975). A similar option (simple cubic lattice) is shown in Fig. 6 (but with approach II, see below).

The above approach (" $I$ ") uses the following definition of the number of steps, $t$. Since in the real crystal a low probability jump occurs after more time, we switch to the time domain, and $t$ now signifies the total time (average) taken up by the most probable jumps, in the hypothetical absence of less probable jumps. For example, a next-nearest ncighbor jump with half the probability of the nearest neighbor jump consumes two "steps" (the time domain is simulated by a step domain) even though it is still a single jump. Here $t$ stays constant $\left(2 \times 10^{5}\right)$. This treatment (" $Y$ ") of introducing next nearest neighbor jumps with reduced probabilities and simultaneously increased jump-times appears justified for the situations 


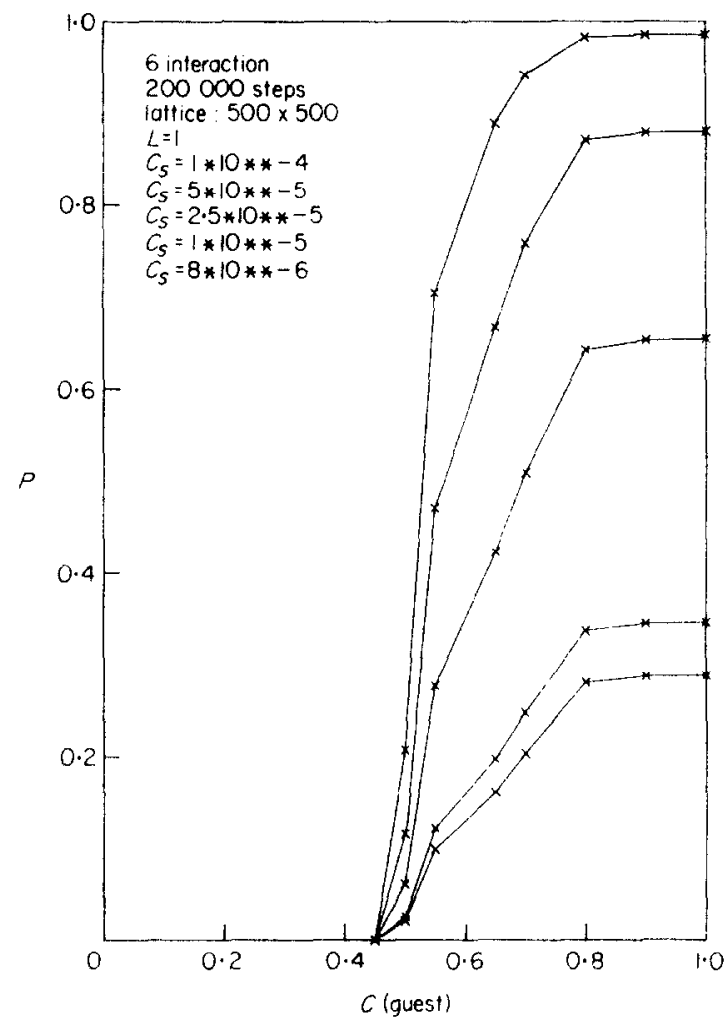

FIG. 4. Registration probability $P$ vs. guest concentration $C_{G}$ with sensor concentration $C_{s}$ as parameter. This is the same as in Fig. 2, except that the topology here is quasitriangular, i.e. two next-nearest neighbor interactions are added to the four nearest ones, with $M^{\prime}=\left(\frac{1}{2}\right) M$ and $p^{\prime}=\left(\frac{1}{2}\right) p$. Note that one $M^{\prime}$ type jump consumes two steps.

where the next-nearest jumps mostly connect guest sites which are not connected by nearest neighbor jumps (and where the next-next-nearest jumps mostly connect sites not connected by nearest and next-nearest ones, etc.). This situation occurs at the lower guest concentrations, i.e. those below and about the critical percolation concentration $C_{C}^{s}$ that is based on the nearest neighbor topology (or the next-nearest topology, respectively, etc.). This happens to be just that $C_{G}$ region where such less probable jumps extend the range of the random walk significantly (below or about the $C_{c}^{s}$ of the nearer interactions). On the other hand, at the higher guest concentrations, well above the nearest neighbor percolation point $C_{C}^{s}$, the jump-times should all be single "steps", as the same time span can be 


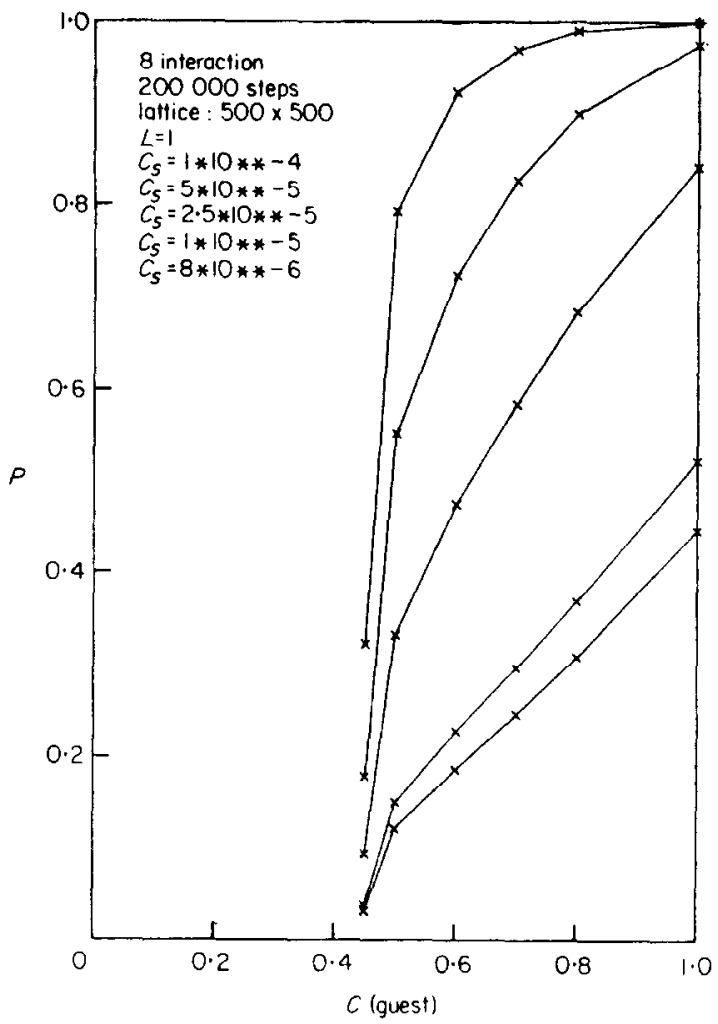

FIG. 5. Registration probability $P$ vs. guest concentration $C_{G}$, with sensor concentration $C_{S}$ as parameter. Same as in Fig. 4 , except that the topology here is quasi-square $(1,2)$ with two third-nearest neighbor interactions added, with $M^{\prime \prime}=\left(\frac{1}{b}\right) M$ and $p^{\prime \prime}=\left(\frac{1}{b}\right) p$. Note that one $M^{\prime \prime}$ type jump consumes five steps.

utilized as well by nearest neighbor jumps. Also, there is a problem of quantum mechanical interference effects, whenever there are two or more channels leading from the old site to the same new site (Schiff, 1955; Blinder, 1974). However, at these higher $C_{G}$ concentrations, where nearest neighbor jumps are very efficient, the relative contribution of the longer jumps is small enough so that any such correction should be small. We also note here a major topological factor working in our favor. This is the particular naphthalene (Hanson, 1970; Hong \& Kopelman, 1971; Ochs \& Kopelman, 1977; Kopelman, 1975; Hartman, 1963) crystal topology which includes four nearest neighbor interactions, compared to only two for any important (translational) next nearest neighbor interactions. We note, however, that 


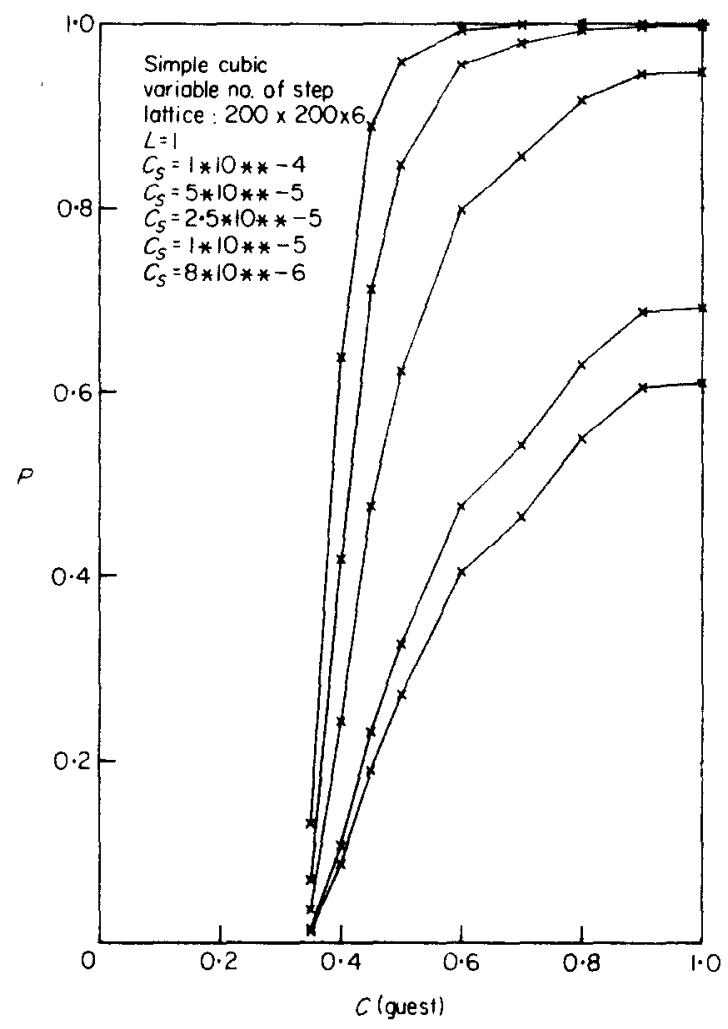

Frg. 6. Registration probability $P$ vs. guest concentration $C_{G}$, with sensor concentration $C_{S}$ as parameter. Same as in Fig. 2 (and 3) but with the following exceptions: (1) The topology is quasi-simple-cubic, with two next-nearest-neighbor interactions, added in the out-of-plane direction, having $p^{\prime}=\left(\frac{1}{3}\right) p$. (2) The total number of steps is $211000 \times C_{\mathrm{G}}$ (compare Fig. 1). (3) Six layers were used to give $N=200 \times 200 \times 6=240000$.

the high probability factors contribute little in those $C_{G}$ regions where few of the pertinent high probability bonds exist. Finally, we would like to emphasize that, when there is no complete coherence, the time an exciton spends on a lattice site is a function of the number of nearest neighbors, next-nearest neighbors, etc. This time decreases, on the average, with increasing guest concentrations. Simulations can be done where each guest site is assigned an exciton occupation time and relative probabilities for the different avenues leading away from it. The exact scheme and its results are reserved for a separate publication. However, the following approximate scheme (approach " $I I ")$ is expected to resemble it closely, using average site occupation times and average directional probabilities. 


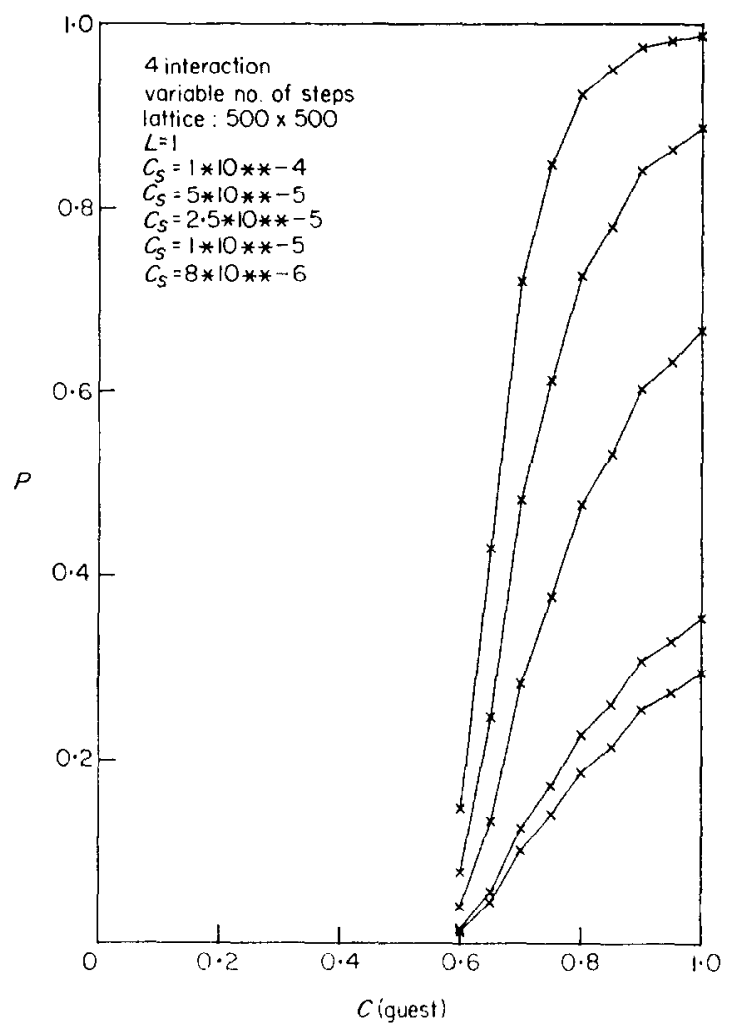

Frg. 7. Registration probability $P$ vs. guest concentration $C_{G}$, with sensor concentration $C_{S}$ as parameter. Same as in Fig. 2, except that the total number of steps is $172000 \times C_{G}$.

In approach II we pay closer attention to the proper meaning of a "jump-time", i.e. the time the excitation spends on the given site until it jumps away (the actual jumping not consuming any time). Following a generalized "golden rule" (Hanson, 1975; Schiff, 1955; Blinder, 1974) we use for the jump-time the expression

$$
t_{j}=\beta^{-1},
$$

where $\beta$ is in units of $\mathrm{Hz}$ and is given by

$$
\beta=\sum_{v} M_{\mu m}
$$

where $M_{\mu \nu}$ is the interaction of the given site $\mu$ with any site $v$. In our particular case we can write, for the pure crystal,

$$
\beta\left(C_{G}=1\right)=4 M+2 M^{\prime}+2 M^{\prime \prime} \text {, }
$$

where $M$ is the nearest neighbor interaction, $M^{\prime}$ the next nearest one and $M^{\prime \prime}$ 


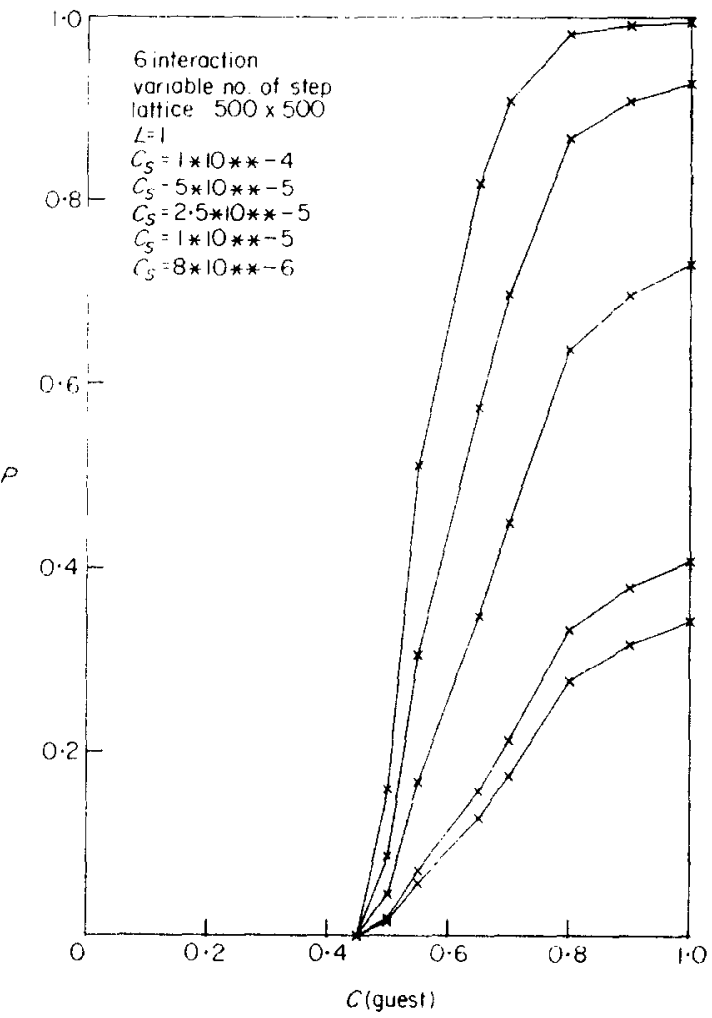

Fig. 8. Registration probability $P$ vs. guest concentration $C_{G}$, with sensor concentration $C_{S}$ as parameter. Same as in Fig. 4, except that the total number of steps is $211000 \times C_{Q}$ and that any jump consumes only one step.

the third nearest one. We also have to assign values to $p: p^{\prime}: p^{\prime \prime}$, the respective probabilities of a nearest neighbor jump, a next nearest one and a third nearest one. We note that for the mixed crystal we write:

$$
\beta\left(C_{G}\right)=C_{G}\left(4 M+2 M^{\prime}+2 M^{\prime \prime}\right),
$$

while the relative probabilities $p^{\prime} / p$ and $p^{\prime \prime} / p$ are preserved.

We use the following values (Hanson, 1970; Hong \& Kopelman, 1971; Ochs \& Kopelman, 1977; Kopelman, 1975): $M=18 \mathrm{~cm}^{-1}, M^{\prime}=8 \mathrm{~cm}^{-1}$, $M^{\prime \prime}=2 \mathrm{~cm}^{-1}$ to esimate $\beta$ and thus $t_{j}$ and $t$. We notice that in approach "II" the total number of steps, $t$, is a linear function of guest concentration:

$$
t=\tau / t_{j}=\tau C_{G}\left(4 M+2 M^{\prime}+2 M^{\prime \prime}\right),
$$

where $\tau$ is the exciton lifetime and again the pairwise interactions ( $M$ etc.) are given in $\mathrm{Hz}$. Figures $7-9$ are based on the above parameters, while 


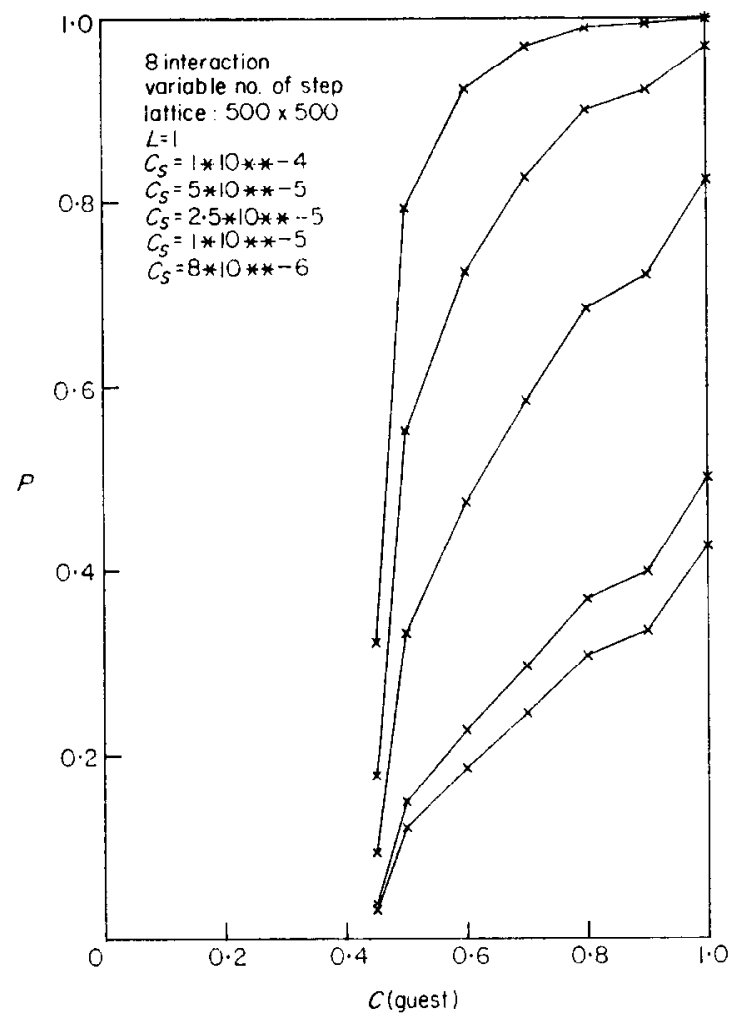

FIG 9. Registration probability $P$ vs. guest concentration $C_{a}$, with sensor concentration $C_{S}$ as parameter. Same as in Fig. 4, except that the total number of steps is $220000 \times C_{G}$, and that any jump consumes only one step.

Fig. 6 was based on $M^{\prime}=6 \mathrm{~cm}^{-1}$. We have neglected here the slight variation in the effective pairwise interaction with concentration (Kopelman et al., 1977b) (due to superexchange [Hong \& Kopelman, 1971]).

Figure 10 shows results for the case where the coherency $l=10$ is the most probable value, in a gaussian distribution with a standard deviation $d=3.0$. Every other parameter is identical to its value in Fig. 2. Comparing this to the $l=1$ case we observe that at high $C_{G}$ concentrations $\left(C_{G}>0.8\right)$ the correlated ("long") jumps provide a more efficient type of motion, while at intermediate concentrations (about $C_{G} \sim 0.7$ ) the opposite happens. Since the random walker is not allowed on $B$ sites, it turns out that for a small number of $B$ sites (high $C_{G}$ ), the correlated (long) jumps enhance the efficiency of motion, the same as in a pure crystal, but when a multitude of $B$ sites is present, the walker encounters more difficulties with long 


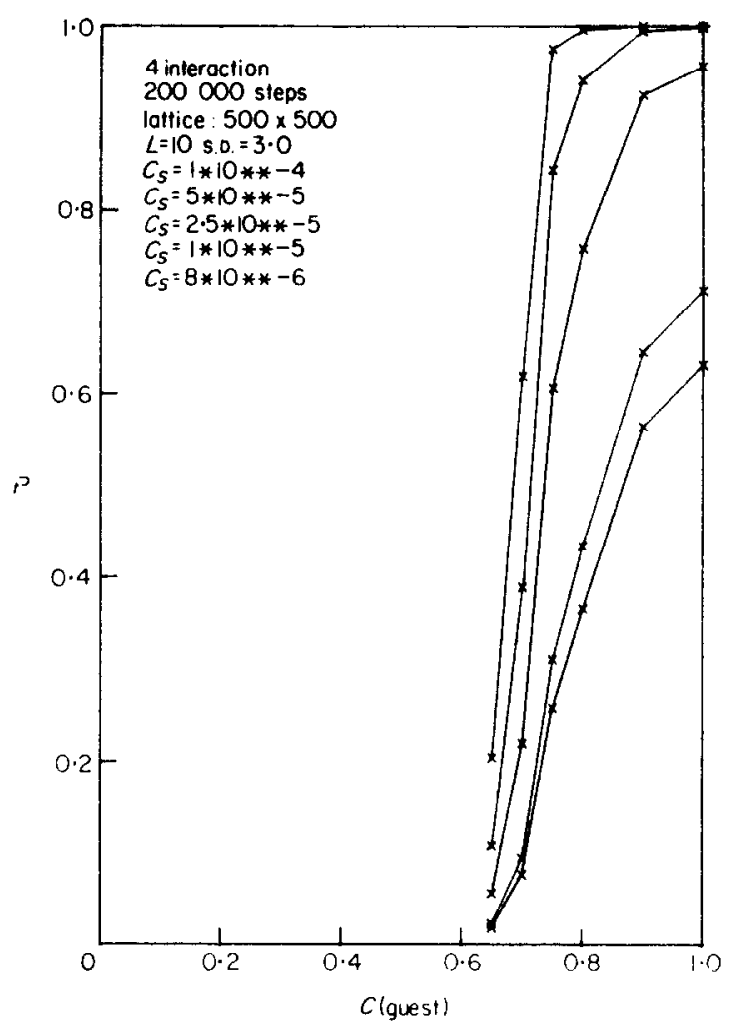

FIG. 10. Registration probability $\boldsymbol{P}$ vs. guest concentration $C_{G}$, with sensor concentration $C_{S}$ as parameter. Same as in Fig. 2, except that the walk is semicoherent, i.e. with directional memory $(L)$ over $l$ steps, where $l$ equals 10 , but with a standard deviation (s.D.) of $d=3.0$. After these $l$ steps, all memory is lost. If the walker "hits" a host site (scatterer) after less than $l$ steps, all memory is lost too.

(correlated) jumps, as it has to penetrate a maze of labyrinth-like narrow passages. This result is shown more dramatically in Figs 11 and 12, where $l=25$ and $l=50$, respectively. The family of curves in the later case gives nearly a limiting behavior, as for $l=100$ (or higher) roughly the same curves are obtained (cf. Figs 13 and 14).

We have also investigated the case where $l$ remains constant throughout the motion $(d=0$, no statistical distribution of $l)$. Our results consistently showed that this type of motion is much less efficient than the cases discussed above. The $C_{G}$ region where $\bar{P}_{\infty}$ approaches unity (Hoshen et al., 1977; Kopelman, 1976 i.e. roughly $C_{G}=0.8$ for the square lattice, appears to be the "turning-point", where coherence becomes conducive to 


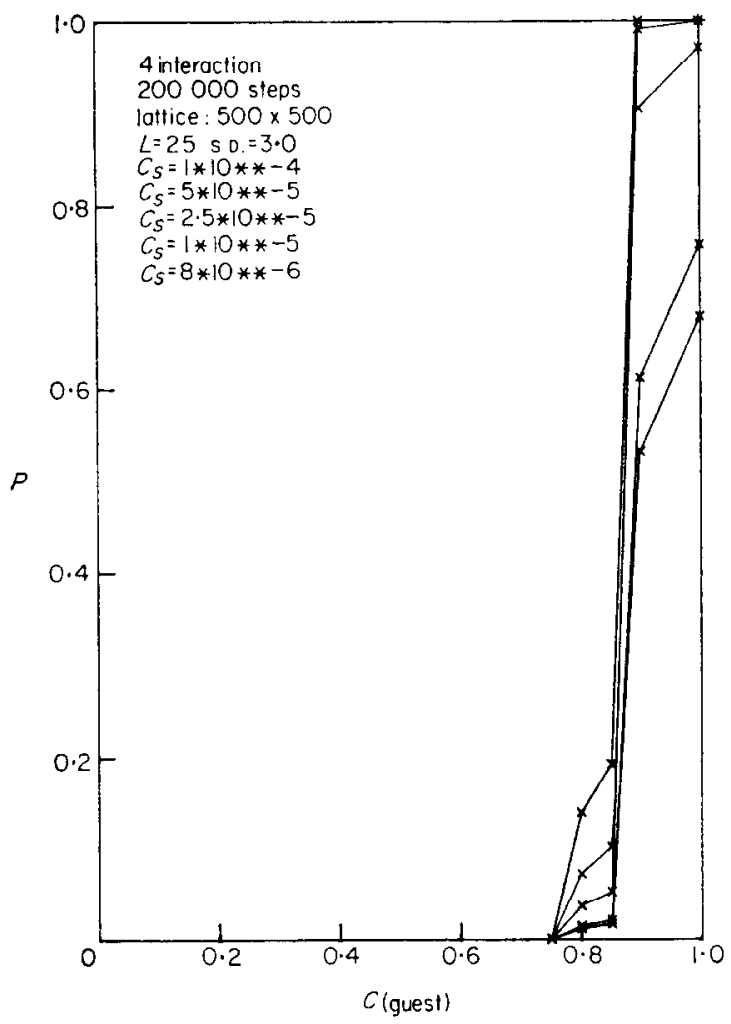

FIG. 11. Registration probability $P$ vs. guest concentration $C_{G}$, with sensor concentration $C_{S}$ as parameter. Same as Fig. 10, except that $l=25$.

the visitation efficiency, i.e. to long range exciton transfer. The following example is intended to show the effect of changing the coherency of the jumps. The lattice of Fig. 15 was constructed in the computer memory. The walker is positioned in the middle of the large square and allowed a random walk motion until it reaches the small square, having to go through the zig-zag passage. A total of 20000 steps was allowed in each case. If the walker, after 20000 steps, never finds the small square, the run is terminated. In Table 1 results are given for 100 runs of each kind. This example shows how inefficient the "long" jumps are for $d=0$, i.e. when there is no distribution. It also shows that between short jumps ( $l=1$, no distribution) and distributed long jumps $(l=10, d=3 \cdot 0)$ the short jumps are more effective when it comes to narrow passages. Obviously this behaviour is important at and below $C_{C}^{s}$, bit does not apply to high values of $C_{G}$ (say for $C_{G}=0.8$ or above), where instead of a maze-like "wood" the random walker 


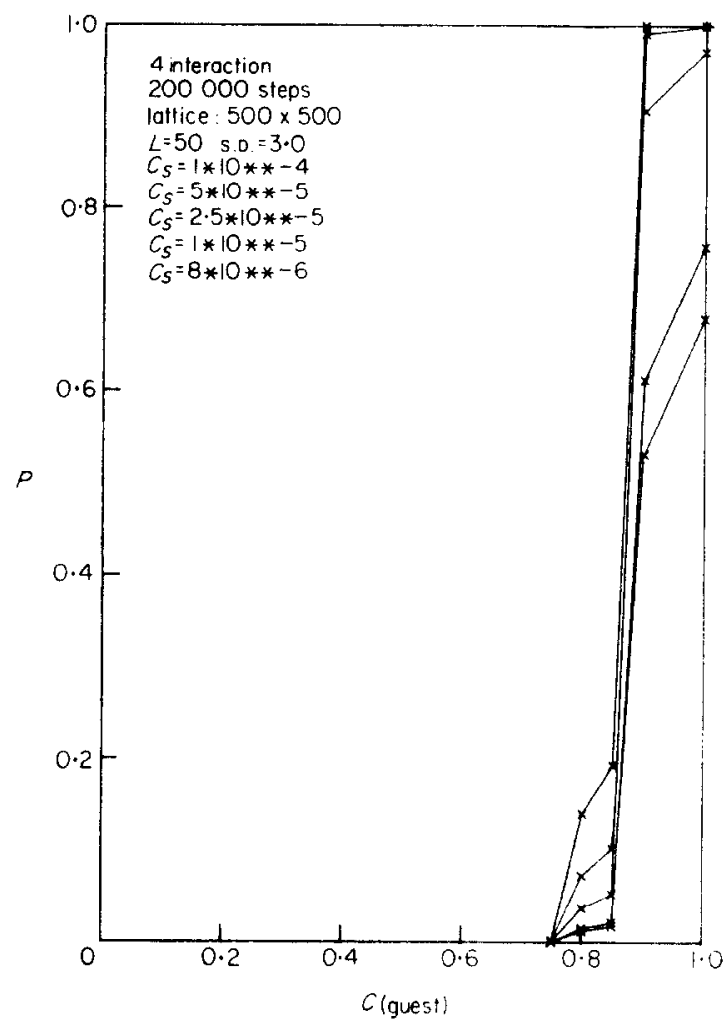

FIG. 12. Registration probability $P$ vs. guest concentration $C_{G}$, with sensor concentration $C_{S}$ as parameter. Same as in Fig. 10, except that $l=50$.

rather finds an "open field" with just a few obstacles. The relative advantages of random walk, compared to coherent motion, in a lattice with a given concentration of "antitraps", has been shown before only for 1-dim. lattices (Hemenger et al., 1972). These may be more significant for energy transfer in 2 and 3-dim. disordered molecular aggregates, synthetic and biological, as appears to be the case for both the exciton migration in isotopic mixed naphthalene (Argyrakis \& Kopelman, 1977; Kopelman, 1976c) and in the photosynthetic units of higher plants (Kopelman, 1976b,c).

\section{Discussion}

The question often asked, relating to the primary process of photosynthesis (Knox, 1975; Sauer, 1975; Olson et al., 1976) is (Montroll, 1969): How long does it take, on the average, until an exciton finds a "trap" (active center)? 


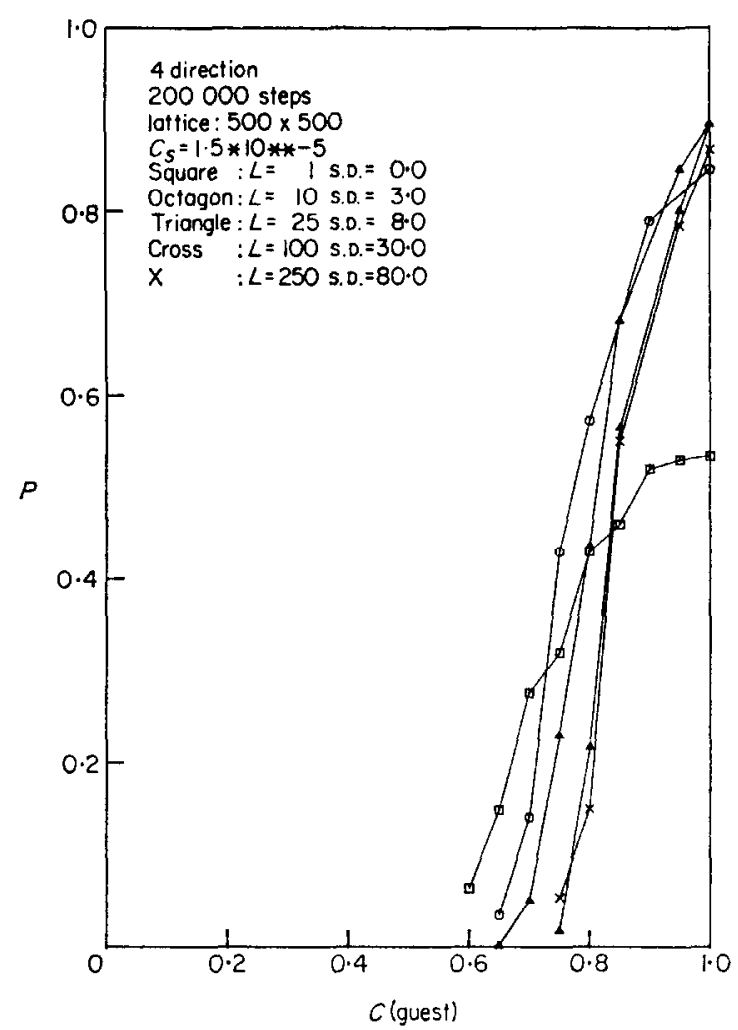

FIG. 13. Registration probability $P$ vs. guest concentration $C_{G}$, with the coherency as a parameter, for a square lattice topology. The sensor concentration $C_{S}$ is $1.5 \times 10^{-5}$. The most probable coherency $l$ varies ( $L$ from 1 to 250 ), while its standard deviation (s.D.) is put at $d=0.3 l$ (rounded to the nearest integer). The lattice size is $500 \times 500$ and the total number of steps $t$ is 200000 . A hop of length $l$ consumes $l$ steps. The origin of the walker is at the center. The results for the highest $l$ values $(100,250)$ are suspect (at high $C_{G}$ values) because of the finite size of the lattice.

This question has been related to the mean first passage time (Montroll, 1969). This formulation partially ignores the finite lifetime of the exciton, as the averaging process includes walking times approaching infinity (Montroll, 1964; Montroll \& Weiss, 1965; Montroll, 1969; LakatosLindenberg \& Shuler, 1971; Lindenberg, 1974; Rosenstock, 1970). Thus, often, a more meaningful question to ask is: What is the probability of an exciton to reach a "trap" (active center) within its lifetime? This question is directly related to the average number of distinct sites visited after a given number of steps. We note that only for simple models (Montroll, 1964; 


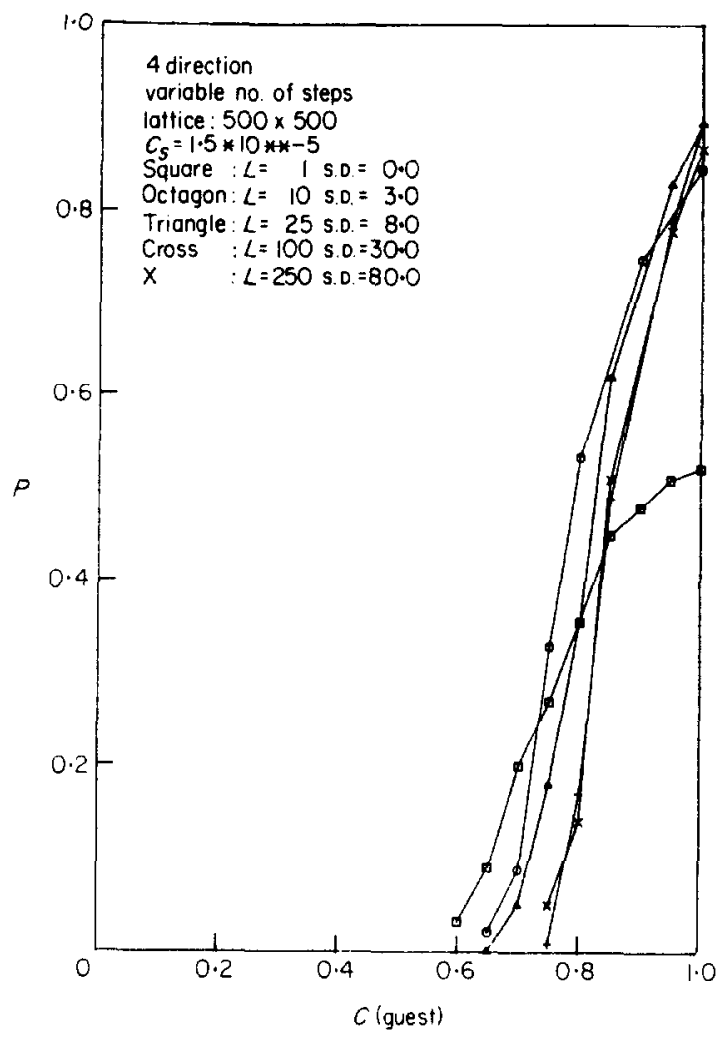

FIG. 14. Registration probability $P$ vs. guest concentration $C_{G}$, with the coherency $(l, d)$ as a parameter. Same as in Fig. 13, except that the total number of steps is $200000 \times C_{O}$.

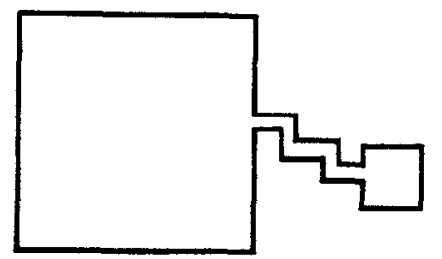

FIG. 15. Scheme of computer simulation for random and semicoherent walk through a small "labyrinth" (each part of the zig-zag is of length four and width one) connecting a $16 \times 16$ square lattice with a $4 \times 4$ one. The walker starts at the center of the large square. Table 1 gives its arrival frequency and time for various coherency parameters $(l, d)$. See text. 
TABLE 1.

Computer game of Fig. $15(\mathrm{t}=20000)$

\begin{tabular}{lcc}
\hline Total: 100 Runs & $\begin{array}{c}\text { Number of times } \\
\text { arrived }\end{array}$ & $\begin{array}{c}\text { Mean number of steps } \\
\text { for arrival }\end{array}$ \\
\hline$l=1, d=0.0$ & 93 & 4339 \\
$l=10, d=3.0$ & 81 & 7339 \\
$l=10, d=0.0$ & $2 \dagger$ & $47 \dagger$ \\
\hline
\end{tabular}

$†$ Note that in these rare runs ending in arrival (two out of 100), arrival happens very "fast".

Montroll \& Weiss, 1965; Montroll, 1969; Lakatos-Lindenberg \& Shuler, 1971, Lindenberg, 1974; Shuler et al., 1976) is there a clear relation between these two questions (see later). We thus calculate the average number of distinct sites visited for given numbers of steps $(t)$, topologies of interactions, crystal sample sizes (with cyclic boundary conditions), and for various guest concentrations (above the critical percolation concentration). We can make a meaningful comparison to existing theories (Montroll, 1964; Montroll \& Weiss, 1965; Montroll, 1969; Lakatos-Lindenberg \& Shuler, 1971; Lindenberg, 1974), by focusing on the number of distinct sites $S_{t}$, visited after $t$ steps, on perfect $\left(C_{G}=1\right)$, infinite or finite (Shuler et al., 1976) lattices with simple (nearest neighbor) topologies. Our computer simulations do agree with the analytical formulas (Montroll, 1964; Montroll \& Weiss, 1965; Montroll, 1969; Lakatos-Lindenberg \& Shuler, 1971; Lindenberg, 1974; Shuler et al., 1976) for the simple cubic lattice (i.e. for $t=40000$; $N=250000$ ) and the 1-dim. one, i.e. for $t=200000, N=250000$, provided that the number of steps $(t)$ is smaller than the lattice size (to avoid complications due to the boundary conditions). However, the twodimensional (square lattice) (Montroll, 1964; Montroll \& Weiss, 1965; Montroll, 1969; Lakatos-Lindenberg \& Shuler, 1971; Lindenberg, 1974) analytical solution for $S_{t}$ is hard to simulate, as the asymptotic formula (Montroll, 1964, 1969; Montroll \& Weiss, 1965)

$$
n \equiv S_{t} \sim \pi t / \ln t
$$

demands a larger $t$ than we can afford to use, if we keep the size of the lattice $(N)$ large compared to the number of steps, i.e. $N>t$. We note that equation (1) agrees with (Appendix A):

$$
P=1-\left(1-C_{S}\right)^{n} \text { iff } n \ll N \text { and } C_{G}=1,
$$

where the index $m$ is omitted as the whole crystal is one "cluster". We also note here that we checked the random generation routine URAND against 
a Tausworthe random number generator (Hoshen, 1976; Tausworthe, 1965; Whittlesey, 1968) and found, within our precision, complete consistency. We also checked our routine computer program against a second one, which we wrote for one-component lattices, and found complete consistency.

Our results, based on equation (1) and our computer simulations, cannot be tested against the analytical solutions Montroll (1969) has given for the first passage time, not even for the where $C_{G}=1$, i.e. no $B$ component, and where also the interaction topology is that of a square or triangular lattice with equivalent nearest neighbor bonds. The first passage time is defined (Montroll, 1964; Montroll \& Weiss, 1965; Montroll, 1969; LakatosLindenberg \& Shuler, 1971; Lindenberg, 1974) as the average number of steps $\langle t\rangle$ required to reach for the first time a given site $s$, when the walker is originally on the origin site, for a finite lattice of size $\bar{N}$ (with cyclic boundary conditions). If the site $s$ is far enough from the origin, and the size $\bar{N}$ large enough, the solutions are (Montroll, 1964; Montroll \& Weiss, 1965; Montroll, 1969; Lakatos-Lindenberg \& Shuler, 1971; Lindenberg, 1974):

$$
\begin{gathered}
\langle t\rangle=\pi^{-1} \bar{N} \ln \bar{N}+0.195056 \bar{N}+\text { higher order terms } \\
\text { iff square lattice, } \\
\langle t\rangle=(2 \pi)^{-1} 3^{\frac{1}{2}} \bar{N} \ln \bar{N}+0.235214 \bar{N}+\text { higher order terms } \\
\text { iff triangular lattice. }
\end{gathered}
$$

In a lattice with $G / Z \equiv \bar{N}$, the definition of $\langle t\rangle$ is apparently based on a probability of unity $(P=1)$ for finding any such site after $\langle t\rangle$ steps, and thus a probability of unity for having visited all $\bar{N}$ sites of the lattice within these $\langle t\rangle$ steps. To compare our results with such a prediction would be a tall order. No matter how many computer experiments are averaged over, the simulation will give $P=1-\delta$. It is not entirely clear what portion of $\delta$ will be due to fluctuations and which one due to the inherent contradiction in terms (Montroll, 1964, 1969; Montroll \& Weiss, 1965; Lakatos-Lindenberg \& Shuler, 1971; Lindenberg, 1974). Given the uncertainty in $\delta$, we note that a simulation with $\alpha\langle t\rangle$ steps, where $\alpha$ may vary from 0.9 to 1.00 , will provide results for $P$ that look equally "acceptable". This is so because the above simulation is done with a finite number of steps (of order $\langle t\rangle$ ). On the other hand, we cannot afford simulations where an unspecified and unlimited number of steps, $t$, is spent each time until an arbitrary site $s$ is visited (or, alternatively, until all sites are visited), followed by averaging over all these $t$ values to give $\langle t\rangle$.

Obviously, Montroll's formalism (Montroll, 1969) is not applicable for our binary lattice problem, i.e. where $C_{B} \neq 0$. Even for $C_{B}=0$ our simulations, for the triangular and square $(1,2)$ lattices, usually involve two or three unequal kinds of bonds (interactions) thus providing results outside 
the domain covered by equations 9-12. Moreover, our simulations for non-trivial coherencies $(l>1)$ are also outside the domain of equations 9-12, and the only applicable analytical solutions have been derived (Lakatos-Lindenberg \& Shuler, 1971; Lindenberg, 1974) for pure, linear lattices.

The following trends are observed in our simulations:

(1) Topology: As the number of bonds increases, the random walk becomes more "efficient". While this is expected even for $C_{G}=1$ (see above) it is more important for lower concentrations, and especially close to the critical percolation concentrations. For instance, below the square lattice critical concentration (0.593) we expect no long-range random walk for a square lattice, i.e. $P \rightarrow 0$, but we do expect finite probabilities, i.e. $P>0$, for the triangular [or square $(1,2)$ ] lattices, as long as $C_{G}$ is above the triangular lattice critical site percolation of 0.5000 (or the square $(1,2)$ one of 0.41 ).

(2) "Mixed" Topologies: Next nearest bonds with reduced probabilities (see above) influence the efficiency especially at lower $C_{G}$, where they "push down" the critical percolation concentration (i.e. Fig. 5), even in the case where significantly reduced probabilities are allocated to some of the weaker bonds. This result is true whether the weaker bond jump is assigned the usual jump-time or a longer one (cf. Fig. 9). However, the more time (number of steps) taken up by these jumps, the lower is the transport efficiency. This last point becomes obvious if one considers the limiting case where some very weak bonds are assigned a finite, though very small, jumping probability, concomittantly with a jump-time that is long enough to approach the total lifetime, i.e. the total number of steps $(t)$.

(3) Number of steps (t): More random steps give a higher probability $P$, i.e. Fig. 3, except below the critical percolation concentration $\left(C_{C}^{s}\right)$ or in the case of supertransfer (see earlier).

(4) Number of Sensors ( $Z$ ): The lower the sensor (supertrap) concentrations $C_{S}$, the lower the registration probability, i.e. Fig. 4. At very low $Z$, i.e. $C_{S} \leqslant n_{m}^{-1}, P$ is linear with $Z$. However, again, at very high $C_{S}$ we get "supertransfer" and $C_{S}$ has little effect (Fig. 3 of paper 1 [Hoshen \& Kopelman, 1976]). Also, below the critical guest concentration there is little long-range transport, irrespective of the value of $C_{S}$ (however, this is not true for short-range transport [Kopelman et al., 1977b; Kopelman, $1976 c]$ ).

(5) Coherency (l): The effect of the coherency $l$ is most interesting. As already pointed out above, a larger coherency favors the long-range transport at higher $C_{G}$ values but hinders it at lower values that are still well above the critical concentration (below it, it obviously makes little difference). 
The reason for this turning point has already been discussed above. The effect of the parameter $l$ is probably the most important result of this study. In our physical systems it expresses the coherence of the excitonic motion (Kenkre \& Knox, 1976; Silbey, 1976; Pearlstein, 1972; Hemenger et al., 1972; Lakatos-Lindenberg, 1972; Hemenger et al., 1974). Our model was chosen with this idea in mind, and it gives a direct handle for coherence studies (Argyrakis \& Kopelman, 1977; Kopelman, 1976b,c). However, one has to consider the dynamics of the system very carefully. Impurities, dislocations, exciton-phonon scattering, trapping and detrapping, the effects of temperature, all have to be taken into account (Kopelman et al., 1977a, b). We reserve this for a future study.

(6) Coherency distribution (d): The standard deviation $d$, of the coherency $l$, plays an important role as well. As expected intuitively, a small $d$ (i.e., $d=0$ ) amplifies the effect of the $l$ parameter at the "lower" $C_{G}$ concentration, i.e. below $0 \cdot 8$, cf. Figs 11 and 13 for $l=25$. However, an unreasonably high $d$ value, i.e. $d=l$, may give results closer to those of $l=1$.

Most of our given simulation examples involve two-dimensional lattices. These are not necessarily cheaper to simulate, as one sometimes needs larger two-dimensional lattices than three dimensional ones to get significant results (Argyrakis \& Kopelman, unpublished). Even though there are basic differences between the two and three dimensional case, both in the percolation problem (Hoshen et al., 1977) and stochastic one, we expect all the trends demonstrated here to be valid for three-dimensional lattices. The effects of $C_{G}$ and $C_{S}$ are already shown in Fig. 6.

We note from Figs 13 and 14 that for the one-component (pure) crystal, at high $l(25-250)$, the number of distinct sites visited approaches the theoretical limit (Appendix B) of:

$$
n_{m} \rightarrow 2 t / 3 \text { (square lattice, } 4 \text { directions), }
$$

where we obey the theoretical condition:

$$
N \gg t \gg l \gg d \gg 1 \text {. }
$$

However, the above limit is based on a very large lattice. Obviously, our results are already suspect for $l=250$, when our lattice size $N$ is $500 \times 500$. This prevents us from getting meaningful results for higher coherency (l) values (for one-component or high $C_{G}$ lattices).

Our simulations are performed only for lattices with $C_{G}>C_{C}^{s}$. This guarantees that our arbitrarily "landed" walker finds itself on the maxicluster. Our formalism is geared (Kopelman, 1976c) toward excluding the very improbable case of "landing" on a minicluster. In practice we never had such an improbable happening. We thus have an approach that eliminates huge statistical fluctuations in $n_{m}$. This has been discussed in 
greater detail elsewhere (Kopelman, 1976c). We just note that for $C_{G}<C_{C}^{s}$ one obviously gets severe fluctuations in $n_{m}$. Therefore, we do not report results for the latter regions. However, instead of simulation, one should simply use (Kopelman, 1976b) the formula:

$$
P=(Z / G) I_{A v}^{\prime} \text { iff } C_{G}<C_{C}^{s},
$$

where $I_{A V}^{\prime}$ is the reduced average cluster size (Hoshen \& Kopelman, 1976; Hoshen et al., 1977). As mentioned above, this term should also be added for $C_{G}>C_{C}^{s}$, but in our case of very low $Z$ this correction in negligible, except at $C_{G} \approx C_{C}^{s}$, a region we avoided, where equation (15) has to be replaced by a better approximation [see papers I (Hoshen \& Kopelman, 1976) and II (Kopelman et al., 1977b)].

Finally, our most important result seems to be the switching from a mostly ordered regime, where "the more "coherent" the motion, the more efficient it is in visiting lattice sites' to a disordered regime where 'the more "random" the motion, the more efficient it is in visiting lattice sites'. In our specific simulations on a random binary lattice, this switching occurs at about a guest concentration of 0.8 (Figs 13 and 14). This implies to us that one should not "worry" about the ambient temperature kT being large enough to cause significant exciton-phonon coupling and thus lead (Knox, 1975; Silbey, 1976) to incoherent exciton transfer in biological aggregates. If such aggregates are inhomogeneous enough, such incoherent or random energy transfer may well be the most optimal mode of transfer. Indeed, the recent estimates by Swenberg et al. (1976) show that such random walk in a model photosynthetic system is fast enough to reach the active center ("supertrap") before significant losses due to fluorescence can occur.

We also note that the simulations described in detail in this work led to our (Kopelman, 1976b,c) photosynthetic model which rationalizes the ratio of antenna to active-center sites usually found in the light harvesting systems of green plants. This model showed that there is little gain, and possible loss, for a ratio $>10^{3}$, while there is a definite advantage for ratios $\gtrsim 10^{2}$.

\section{Conclusions}

We have simulated stochastic percolation for a range of topologies, concentrations and random walk parameters. The latter are based on what we believe to be reasonable physical assumptions for exciton migration in molecular aggregates. In situations where the physical picture is currently cloudy, we have used extreme models, to serve as limiting cases 
and as future guides, indicating how crucial certain physical assumptions are for a given problem. We observed the following patterns:

(1) The time dependent, stochastic percolation behavior approaches smoothly the time independent supertransfer limit for all the models studied.

(2) For lattices with low scatterer concentrations, semicoherent stochastic motion has a high visitation efficiency relative to incoherent motion, as intuitively expected, but the opposite is true for high scatterer concentrations. The turning point is shown to be significantly above the critical percolation concentration. Thus we are dealing here with a non-trivial effect of great significance, i.e. incoherent walk being more efficient than coherent one in many molecular aggregates.

(3) The effects of topology follow the intuitively obvious pattern: The larger the co-ordination number (the number of bonds), the higher the visitation efficiency. The same goes for the number of steps and for the number of sensors in the ternary lattices. However, the above statements are true only above the critical percolation concentration (for the given topology) and outside the "supertransfer" limit).

(4) The effects of the coherency distribution ("standard deviation" $d$ ) are far from trivial. The higher $d$ (for a fixed coherency $l$ ), the higher the visitation efficiency, in a binary lattice with a significant number of scatterers. However, even without scatterers this efficiency is increased going from $d=0$ to $d \gg 1$.

We find the effects of conclusion 4 to be of most interest to the energy transfer in both synthetic "model systems", mixed crystals of naphthalene, and in disordered, inhomogeneous biomolecular aggregates, which the photosystems I and II might well turn out to be.

We thank Dr Joseph Hoshen for assistance with the programming and for very helpful discussions. We also thank Eric $M$. Monberg for supplying the necessary $\bar{P}_{\infty}$ values "hot off the press".

\section{REFERENCES}

AвOOD, L. G. (1976). Basic Neurochemistry, (G. Siegel ed. et al) Boston Little Brown, p. 103.

Argyrakis, P. \& Kopelman, R. (1977). J. chem. Phys. 66, 3301.

ARgYrakis, P. \& Kopelman, R. (unpubl.).

Blinder, S. M. (1974). Foundations of Quantum Dynamics, Ch. 7, Academic Press: London.

Borisov, A. Y. \& Godik, V. I. (1973). Biochim. biophys. Acta 301, 227.

Brandt, W. W. (1975). J. chem. Phys. 63, 5162.

Caple, M., Chow, H-C., Burns, R. M. \& Strouse, C. E. (1976). Brookhaven Symp. Biol. $28,56$.

DAYYDOv, A. S. (1973). J. theor. Biol. 38, 559. 
Davydov, A. S. \& Kislukha, N. I. (1976). Phys. Stat. Solidi. b, 75, 735.

Duckers, L. J. \& Ross, R. G. (1974). Phys Lett. 49A, 361.

El-Kareh, T. B. \& Wolf, H. C. (1968). Mol. Cryst. 4, 195.

Hanson, D. M. (1970). J. chem. Phys. 52, 3409.

Hartmann, P. (1963). Physics and Chemistry of the Organic Solid State (D. Fox, M. M. Labes \& A Weissberger, eds). New York: Interscience (Wiley).

Hemenger, R. P., Pearlstein, R. M. \& Lakatos-Lindenberg, K. (1972). J. math. Phys. $13,1056$.

Hemenger, R. P., Lakatos-Lindenberg, K. \& Pearlstein, R. M. (1974). J. chem. Phys. 60,3271 .

Hong, H. K. \& Kopelman, R. (1971). J. chem. Phys. 55, 724.

Hoshen, J. \& Kopelman, R. (1976a). J. chem. Phys. 65, 2817.

Hoshen, J. \& Kopelman, R. (1976b). Phys. Rev. B14, 3438.

Hoshen, J., Kupelman, R. \& Monbero, E. M. (1978). J. Stat. Phys. in press.

KATZ, J. J. (1976). Brookhaven Symp. Biol. 28, 16.

KENKRe, V. M. \& KNOX, R. S. (1976). J. Lumin. 12, 187.

Kohler, M., SCHMid, D. \& Wolf, H. C. (1976). J. Lumin. 14, 41.

KNox, R. S. (1975). Bioenergetics of Photosynthesis (Govindjee, ed.). New York: Academic Press.

Kopelman, R. (1975). Excited States II (E. C. Lim, ed.). New York: Academic Press.

Kopelman, R. (1976a). J. Lumin. 12, 775.

Kopelman, R. (1976b). J. Phys. Chem. 80, 2191.

Kopelman, R. (1976c). Radiationless Processes in Molecules and Condensed Phases, Topics in Applied Physics, (F. K. Fong, ed.), Vol. 15, Berlin: Springer.

Kopelman, R., Monberg, E. M., Ochs, F. W. \& Prasad, P. N. (1975a). Phys. Rev. Lett. 34, 1506.

Kopleman, R., Monberg, E. M., Ochs, F. W. \& Prasad, P. N. (1975b). J. chem. Phys. $62,292$.

Kopelman, R., Monberg, E. M. \& Ochs, F. W. (1977a). Chem. Phys. 19, 413.

Kopelman, R., Monberg, E. M. \& Ochs, F. W. (1977b). Chem. Phys. 21, 373.

LaKatos-Lindenderg, K. \& ShUler, K. E. (1971). J. math. Phys. 12, 633.

Lakatos-Lindenberg, K., Hemenger, R. P. \& Pearlstein, R. M. (1972). J. chem. Phys. $56,4852$.

Lindenderg, K. (1974). J. Stat. Phys. 10, 485.

Montroll, E. W. (1964). Proc. Symp. Appl. Math. XVI, 193.

Montroll, E. W. (1969). J. math. Phys. 10, 753.

Montroll, E. W. \& Weiss, G. H. (1965). J. math. Phys. 6, 167.

Muller-KrumbhaAr, H. (1974). Phys, Lett. 50A, 27.

Ochs, F. W. \& Kopelman, R. (1977). J. chem. Phys. 66, 1599.

Olson, J. M., Ke, B. \& Thompson, K. H. (1976). Biochim. biophys. Acta 430, 524.

Pearlstein, R. M. (1972). J. chem. Phys. 56, 2431.

Pearlstein, R. M. (1966). Brookhaven, Symp. Biol. 19,

Ressler, N. (1969). J. theor. Biol. 23, 425.

RoBinson, G. W. (1966). Brookhaven, Symp. Biol. 19, 16.

Rosenstock, H. B. (1970). J. math. Phys. 11, 487.

SAUER, K. (1975). Bioenergetics of Photosynthesis (Govindjee, ed.). New York: Academic Press.

Schiff, L. I. (1955). Quantum Mechanics, 2nd edn, Ch. VIII. New York: McGraw-Hill.

Shuler, K. E., Silver, H. \& LindenberG, K. (1976). J. Stat. Phys. 15, 393.

Silbey, R. (1976). Ann. Rev. plyys. Chem. 27, 203.

Swenberg, C. E., Dominisanni, R. \& Geacintov, N. E. (1976). Photochem. Photobiol. 24, 601 .

TASAKI, I. (1974). Current Topics in Membranes and Transport (F. Bronner \& A. Kleinzeller, ed.) p. 284. Academic Press, New York. 
TAusworthe, R. C. (1965). Math. Comput. 19, 201.

TIEN, H. T. (1976). Brookhaven Symp. Biol. $28,105$.

UCHIDA, K. \& TOMURA, M. (1974). J. Phys. Soc. Japan 36, 1358.

WhitTlesey, J. R. B. (1968). Comm. $A C M$ 11, 641.

\section{APPENDIX A \\ Extreme Sensor Concentrations}

At very low sensor concentration $C_{S}$, paper I (Hoshen \& Kopelman, $1976 a$ ) gives the simple formula:

$$
F_{n}=C_{S} n_{m} / C_{G}=Z G^{-1} n_{m} \text { iff } F_{n} \ll 1 .
$$

This gives, from equation (3),

$$
P=Z G^{-1} n_{m} \vec{P}_{\infty} \quad \text { iff } \quad C_{S} \ll C_{G} n_{m}{ }^{-1} .
$$

Thus the visitation probability is linear with $Z$, i.e. $C_{S}$, as well as with $n_{m}$ and $\bar{P}_{\infty}$. This accounts for our simulation curves (Figs 2-14) not going below about $C_{S}=10^{-6}$.

The high $C_{S}$ region of about $C_{S}>10^{-3}$ is also not calculated, due to the trivial relationship (Hoshen \& Kopelman, 1976a)

$$
P=\bar{P}_{\infty} \quad \text { iff } \quad C_{G} \gg C_{C}^{s} \gg C_{S} \text { and supertransfer, }
$$

or the more precise equation (Hoshen \& Kopelman, 1976a):

$$
P=\bar{P}_{\infty}+Z G^{-1} I_{A v}^{\prime} \text { conditions as above. }
$$

For $C_{G}$ closer to the critical percolation concentration, we have (Hoshen \& Kopelman, 1976a):

$$
\begin{gathered}
P=\bar{P}_{\infty}+\sum_{m \neq m}^{\prime}\left[1-\left(1-m G^{-1}\right)^{\mathrm{z}}\right] i_{m} m G^{-1} \text { iff } C_{G}>C_{C}^{s} \geqslant C_{S} \\
\text { and supertransfer, }
\end{gathered}
$$

where the summation omits the maxicluster $m^{\prime}$ and $i_{m}$ is the frequency (Hoshen \& Kopelman, 1976b) of cluster $m$. Curves giving $P$ according to equation 5 have been given elsewhere (Hoshen \& Kopelman, 1976a; Kopelman et al., 1977b; Hoshen et al., 1977; Kopelman, 1976c).

Returning to very low sensor concentrations, we notice that the number of distinct sites visited, $n_{m}$ is quasilinear with guest concentration $C_{G}$ (see Fig. 1), i.e.

$$
n_{m} \propto C_{G} \text { iff } C_{G}>C_{C}^{s} .
$$

Using equation (A2) and the trivial relationship $Z G^{-1}=C_{S} C_{G}{ }^{-1}$ one gets

$$
P \alpha C_{S} \bar{P}_{\infty} \quad \text { iff } \quad C_{G}>C_{C}^{s} \text { and } C_{S} \ll n_{m}{ }^{-1} \text {, }
$$

i.e. the visitation probability is linear with both $C_{S}$ and $\bar{P}_{\infty}$. Our lowest $C_{S}$ curves (i.e. $C_{S}=10^{-6}$ on Fig. 2) seem to obey this relationship, within the computation uncertainty. 
The low sensor concentration results agree, for $C_{G}=1$, with the result of equation (10), which is equivalent to

$$
n=\ln (1-p) / \ln \left(1-C_{S}\right),
$$

giving

$$
n=-C_{s}^{-1} \ln (1-P) \text { iff } C_{s} \ll 1 \text {, }
$$

or

$$
P=1-\exp \left(-n C_{s}\right) \text { iff } C_{s} \ll 1 .
$$

This is identical to the result of paper I (Hoshen \& Kopelman, 1976a):

$$
P=F_{n}=1-\exp \left(-C_{S} n\right) \text { iff } N / n \rightarrow \infty,
$$

which gives,

$$
P=n C_{S} \quad \text { iff } \quad C_{S} \ll n^{-1} \ll N^{-1} .
$$

This result is identical with equation (A.2) for $C_{G}=1$.

The high sensor concentration results obviously agree with those of Montroll (Montroll, 1969), as from equation (A.4) one gets

$$
P=1 \text { iff } C_{G}=1 \text { and supertransfer, }
$$

as for $C_{G} \rightarrow 1$ one has $\bar{P}_{\infty} \rightarrow 1$ and $I_{\text {Av }}^{\prime} \rightarrow 0$.

\section{APPENDIX B}

\section{Semicoherent Walk Limit for a Multidimensional Perfect Lattice}

We discuss here a simple limit for a physically reasonable coherent walk in a one-component multidimensional perfect lattice, where the scattering is assumed to be homogeneous, i.e. due to exciton-phonon coupling.

Theorem:

$$
n_{t}^{l} \sim \frac{b-2}{b-1} t \quad \text { if } \quad N \gg t \gg l \gg d \gg 1 .
$$

Here $n_{t}^{l}$ is the number of distinct sites visited after $t$ steps, with a mean directional correlation $l$, a standard deviation $d$, and a lattice of size $N$ with a topology of $b$ bonds (directions) per site.

\section{Proof:}

At the end of the series of $l_{1}$ correlated steps, the walker has a probability of $b^{-1}$ for turning back. It now moves $l_{2}$ steps, but as $d \ll l$,

$$
\left|l_{1}-l_{2}\right| / l_{1} \ll 1
$$

and the walker retraces all previous $l_{1}$ sites, with a small negative deviation 
(on the average). The fraction of sites $X$ revisited due to this factor is thus, in general,

$$
X \lesssim b^{-1}+b^{-2}+\ldots+b^{-t / l} .
$$

As $t \gg l$, we can approximate $X$ by an infinitely descending geometric series:

$$
X \lesssim b^{-1} /\left(1-b^{-1}\right)=(b-1)^{-1},
$$

again with a small negative deviation (on the average). As $l \gg 1$, we assume that after the $i$-th scattering event the walker has a probability of $(b-1) / b$ of escaping the previous $l_{i}$ sites, i.e. never revisiting any of them. This assumption contributes a small positive deviation (on the average) to the fraction of sites visited. We note that the escape clause would be invalid for $d=0$ (confining the walker to a superlattice with lattice constant $l$ ). However, it is reasonable for $d \gg 1$. We can assume that our small positive and negative deviations cancel (on the average) to the extent that the total fraction of sites revisited (once or more) is given by equation (A.4). We thus get the fraction of distinct sites visited:

$$
1-X \sim 1-(b-1)^{-1}=(b-2) /(b-1) .
$$

As the total number of sites visited is $t$,

$$
n_{l}^{l} / t \sim(b-2) /(b-1) .
$$

This obviously gives equation (B.1). Q.E.D. 